\title{
Kangal Köpeklerinde Trachea'nın Bilgisayarlı Tomografi ile Üç Boyutlu Değerlendirilmesi
}

\author{
Ömer ATALAR ${ }^{1 *}$, Mustafa KOÇ ${ }^{2}$, Zait Ender ÖZKAN ${ }^{1}$, Saime Betül BAYGELDi ${ }^{1}$, \\ Yeşim ASLAN KANMAZ ${ }^{1}$
}

${ }^{1}$ Fırat Üniversitesi Veteriner Fakültesi Anatomi Anabilim Dalı, Elazı̆̆, Türkiye.

${ }^{2}$ Fırat Üniversitesi Tıp Fakültesi Radyoloji Anabilim Dalı, Elazığ, Türkiye.

\begin{abstract}
Özet: Bu araştırma; Kangal köpeklerinde ilk kez bilgisayarlı tomografik görüntüler kullanılarak trachea'nın ayrıntılı anatomisini elde etmek amacıyla gerçekleştirilmiştir. Çalışmada 5 erkek ve 5 dişi olmak üzere toplam 10 erişkin Kangal köpeği kullanılmıştır. Multidedektör bilgisayarlı tomografi (MDBT) cihazından elde edilen görüntüler, üç boyutlu (3D) modelleme yazılımı (VITAL, Vitrea 2,HP XW 6400) ile trachea'nın üç boyutlu yeniden yapılandırılmasında kullanılmıştır. Trachea'nın ölçümleri; hem pars cervicalis hem de pars thoracalis bölgelerinde; cranial ve caudal noktalar belirlenerek yapılmıştır. Trachea'nın 4 noktasından alınan transversal ve vertical çap ölçümleri, bu çapların birbirlerine oranı (T/V) ile kesitsel lümen alanları (crosssectional area CSA), BT (Bilgisayarlı tomografi) tarayıcı yazılımı ile ölçülmüştür. Erkekler ve dişiler arasında önemli bir istatistiksel fark bulunmamıştır. Trachea'nın başlangıç ve bitiş kısımlarında çapların arttığı gözlenmiştir. Transversal çapların bütün ölçüm noktalarında vertical çaplardan daha yüksek değerlerde olduğu tespit edilmiştir. Üç boyutlu modelleme ile trachea'nın dorsoventral basık ve tam olmayan elipsoid şeklinde olduğu ortaya konmuştur. Araştırmanın ilk kez yapılmış olması sebebiyle, anatomi alanındaki benzer çalışmalara kaynak teşkil edebileceği düşünülmektedir.
\end{abstract}

Anahtar Kelimeler: Kangal köpeği, Üç boyutlu görüntü, Trachea, Bilgisayarlı tomografi.

\section{Three Dimensional Evoluation of Trachea with Computed Tomography in The Kangal Dogs}

\begin{abstract}
This study was conducted to obtain a detailed anatomy of the trachea in Kangal dogs, for the first time using computerized tomographic images. In this study, 5 male and 5 female total 10 adult Kangal dogs were used. The images obtained from a multidetector computerized tomography device (MDBT) were used for 3D reconstruction of the trachea with the 3D modelling software (VITAL, Vitrea 2, HPXW 6400). The measurements of the trachea were made from the cranial and caudal points determined at the cervical and thoracal regions. Transversal and vertical diameters of the trachea taken from 4 different points, the ratio of these diameters $(T / V)$ and cross sectional areas (CSA), were measured by CT (computer tomography) scanner software. A significant statistical difference was not found between males and females. An increase in the diameter of the trachea at the beginning and terminal parts was observed. It was found that the transversal diameters were greater than the vertical diameters at all measuring points. In the 3D modelling, trachea has been found to be dorsoventrally flat with an incomplete elipsoid form. It is thought that this work, which was first made in Kangal dogs, may be a source for similar work in the anatomy.

Keywords: Computer tomography, Kangal dogs, Three dimensional image, Trachea.
\end{abstract}

\section{Giriş}

Türkiye'nin özel bir ırkı olan Kangal köpekleri, çoban köpeği olarak oldukça değerli bir ırktır. Üzerinde pek fazla anatomik çalışma yapma imkânı bulunamayan bu köpeklerin, ortalama boyları dişilerde $72-77 \mathrm{~cm}$, erkeklerde $77-86 \mathrm{~cm}$, kiloları ise dişilerde 41-54, erkeklerde 50-66 kg civarındadır. Bu özel ırkın anatomisinin detaylandırılmasında en hızlı, güvenli ve doğru metod bilgisayarlı tomografi ve 3 boyutlu modelleme olarak ifade edilmektedir (Atalar ve ark., 2017; Yılmaz, 2017).

Solunum sisteminin çok önemli bir unsuru olan trachea, hayvan türlerine göre anatomik yapısı itibari ile farklılıklar göstermektedir (Dursun, 1996). Köpeklerde de farklı ırk gruplarında, trachea'da yapı değişiklikleri bildirilmektedir (Evans, 1993).
Brachycephalic (kısa başlı) türlerin nispeten dar trachea'ya sahip olduğu radiografik olarak kanıtlanmıştır, fakat Dachshund ve Basset Hound'lar daha geniş bir trachea'ya sahiptir (Dyce ve ark., 2018). Tür ve ırklar arasındaki bu anatomik varyansların; daha ziyade cartilago trachealis'lerin sayısı, şekilleri ve trachea lumen kesit alanlarında gözlemlendiği ifade edilmektedir (Dursun, 1996; Getty, 1975; Nickel, 1979). Teknolojik gelişmeler bilgisayarlı tomografi ve 3D modellerinin geliştirilmesinde de firsatlar oluşturmaktadır. Trachea'nın üç boyutlu bilgisayarlı tomografi (3DBT) görüntüleri; bu organın patolojik koşullarının tespiti ve anatomik yapısının daha doğru tanımlanması için önemlidir. Son zamanlarda birçok alanda olduğu gibi 
veteriner hekimlikte bilgisayarlı tomografi görüntüleri sıkça kullanılmaktadır. Köpeklerde trachea'nın bilgisayarlı tomografisi üzerinde yapılan çalışmalarda, anlaşıldığı üzere her ırkın trachea'sında hem anatomik yapının hemde ölçümlerin farklı olduğu görülmüştür (Dallman ve Brown, 1984; Kara ve ark., 2001). Köpeklerin trachea'larında yapılan araştırmalarda; kongenital stenoz (Burk ve Ackerman, 1996), tümör (Brovida ve Castagnaro, 1992; Carlisle ve ark., 1991), yabancı cisim (Röcken ve Röcken, 1994) ve collaps vakaları gibi spesifik durumlar (Dallman ve Brown, 1984) sıkça bildirilmektedir. Literatürlerde Kangal köpeklerinin trachea'sında bilgisayarlı tomografi ve üç boyutlu modelleme ile biyometrik ölçümleri üzerine yapılmış herhangi bir araştırma bulunmamaktadır. Bu nedenle yapılan çalışmanın kangal köpeklerine ait trachea verilerini ortaya koyması ve anatomi literatürüne katkı sağlaması bakımından önemli olduğu düşünülmektedir.

\section{Materyal ve Metot}

Araştırma; Fırat Üniversitesi Veteriner Fakültesine tedavi amaçlı getirilen, erişkin Kangal köpeklerinden alınan BT görüntülerinin, retrospektif incelenmesi neticesinde yapılmıştır. Çalışmada Bingöl Üniversitesi Hayvan Deneyleri Yerel Etik Kurul'unun 85680299/20 sayılı onayı alınmıştır. Araştırma için 5 dişi ve 5 erkek olmak üzere toplam 10 erişkin Kangal köpeği kullanılmıştır. Materyaller, xylazine (1.1 mg/kg, i.m., Rompun) ve ketaminin (22 $\mathrm{mg} / \mathrm{kg}$, i.m., Ketalar) kombinasyonu ile genel anesteziye alınmıştır.

Fırat Üniversitesi Tıp Fakültesi Radyoloji Anabilim dalında Toshiba Aquilion 64 kesit BT cihazına yüzüstü konumda yatırılan köpeklerin, multi dedektör bilgisayarlı tomografi (MDCT) görüntüleri elde edilmiştir. Bu görüntüler, kVp 120, mAs $150-200$ ve $0.5 \mathrm{~mm}$ paralel kesit kalınlığı, 0.5 $\mathrm{mm}$ rekonstrüksiyon aralığı, çap görüş alanı (FOV: Field of view) $(30 \mathrm{~cm})$ ve aralık değeri 1-1.5 arasında kaydedilmiştir. Dozaj parametreleri ve tarama, standart protokoller çerçevesinde gerçekleştirilmiştir.

Trachea'nın ölçümleri pars cervicalis'inde; cranial nokta 1.cervical omur (C1) izdüşümünden, caudal nokta 6.cervical omur (C6) düzeyinden, pars thoracalis'inde; cranial nokta 1. thoracal omur (T1) düzeyinden, caudal nokta ise 4. thoracal omur (T4) seviyesinden yapılmıştır. Ölçüm alınan noktalardan enine $(T)$ ve dikey (V) çaplar alınmıştır. Bu noktaların lümen kesit alanları (CSA) hesaplanırken; enine (T) ve dikey (V) çapların yarıçapları ve "pi" değeri çarpılarak bulunmuştur. CSA= a.b. $\pi$ formülü; $a=$ transversal yarı çap, $b=$ vertical yarı çap, $\pi=3,14$ şeklinde uygulanmıştır (Kara ve ark., 2001). Aynı zamanda transversal ve vertical çap oranları da ölçülmüştür (T/V). Çekimler ile köpeklerin trachea'sından yüksek çözünürlüklü MDCT görüntüleri elde edilmiştir. Bu eksenel görüntüler DICOM formatında stokladıktan sonra, veriler çalışma merkezine (VITAL, Vitrea 2, HP XW6400) aktarılmıştır. Görüntüler araştırma alanı içinde değerlendirilmiş ve sonuçlar kaydedilmiştir. Öncelikle iki boyutlu (2D) görüntülerden ilgili kısımlar seçilmiştir. Sonrasında ise üç boyutlu (3D) modelleme oluşturulmuştur. Trachea'nın üç boyutlu (3D) modellemesi yapıldıktan sonra, veriler ortalama \pm standart sapma değerleri olarak sunulmuştur. Öncelikle ham değerlerin normal dağılım gösterip göstermediklerini tespit etmek için, Shapiro-Wilk normallik analizi yapılmıştır. Bu analize göre ham değerlerin normal dağılım gösterdiği saptanmıştır. Tüm veriler tek yönlü varyans analizi (ANOVA) ile karşılaştırılmıştır. Ikili karşılaştırmalar içinde post-hoc Tukey testi kullanılmıştır. $P<0.05$ değeri istatistiki olarak anlamlı kabul edilmiştir. Analizler SPSS (Versiyon 21.0) programında yapılmıştır. Terminoloji olarak Nomina Anatomica Veterinaria esas alınmıştır (Nomina Anatomica Veterinaria, 2012).

\section{Bulgular}

Kangal köpeklerinin trachea ölçümleri ortalama değerleri Tablo 1'de; üç boyutlu modellemesi Şekil 1'de, iki boyutlu görüntüleri ise Şekil 2 ve 3'de sunulmuştur. Yapılan trachea ölçümlerinde erkek ve dişi hayvanlar arasında önemli bir istatistiksel fark bulunmadığı gözlenmiştir. Tablo 1'de; 4 farklı bölgeden yapılan ölçüm değerleri ortalamalarında, trachea'nın transversal ve vertical çapları, bu çapların birbirine oranları ve lümen alanları belirtilmiştir. Ölçüm noktaları arasında T/V oranında belirgin farklılıkları tespit edilmiştir. Ayrıca transversal ve vertical çap oranları, trachea'nın son kısımlarında artış göstermektedir.

Tablo 1. Kangal köpeklerinde trachea ölçümlerine ait ortalama \pm standart sapma değerleri.

\begin{tabular}{|c|c|c|c|c|c|}
\hline & Cranial cervical & Caudal cervical & Cranial thoracal & Caudal thoracal & Önemlilik \\
\hline Vertical çap (mm) & $22.19 \pm 1.79^{a}$ & $20.96 \pm 1.45^{a}$ & $26.04 \pm 1.38^{b}$ & $25.56 \pm 1.52^{b}$ & $\mathrm{P}<0.001$ \\
\hline Transversal çap (mm) & $28.20 \pm 1.87^{a}$ & $27.82 \pm 1.95^{a}$ & $28.83 \pm 2.18^{\mathrm{a}}$ & $35.30 \pm 2.15^{b}$ & $P<0.001$ \\
\hline Lumen kesit alanı (CSA, $\mathrm{mm}^{2}$ ) & $483.75 \pm 70.93^{a}$ & $460.10 \pm 63.62^{\mathrm{a}}$ & $583.71 \pm 66.82^{b}$ & $701.04 \pm 75.71^{\mathrm{c}}$ & $P<0.001$ \\
\hline $\mathrm{T} / \mathrm{V}$ oranı & $1.27 \pm 0.02^{\mathrm{a}}$ & $1.33 \pm 0.02^{b}$ & $1.11 \pm 0.03^{c}$ & $1.38 \pm 0.03^{d}$ & $P<0.001$ \\
\hline
\end{tabular}

Aynı satırda farklı harf taşıyan ( $a, b, c, d)$ ortalama değerler arasındaki farklılık istatistiki açıdan önemlidir. 
Transversal çapın bütün ölçüm bölgelerinde aynı noktanın vertical çapından daha uzun olarak tespit edilmiştir. Hem vertical hem transversal çapın en uzun olduğu nokta, trachea'nın pars thoracalis'inde; en kısa olduğu nokta ise pars cervicalis'inin caudal bölümünde ölçülmüştür. Trachea'nın lümen genişliği, CSA hesabına bakıldığında; en geniş olarak pars thoracalis'inin caudal ucunda, en dar olarak ise pars cervicalis'in caudal ucunda gözlemlenmiştir. T/V oranı, bütün bölgelerde 1.00 'in üzerinde ve birbirine yakın değerlerde seyretmektedir.

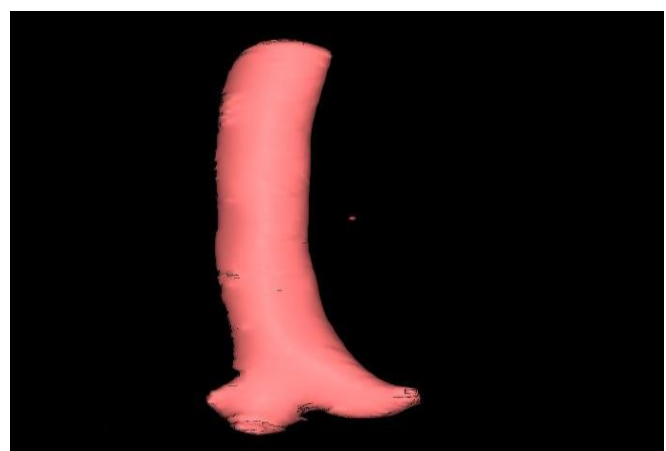

Şekil 1. Kangal köpeği trachea'sının üç boyutlu (3D) modellemesi.

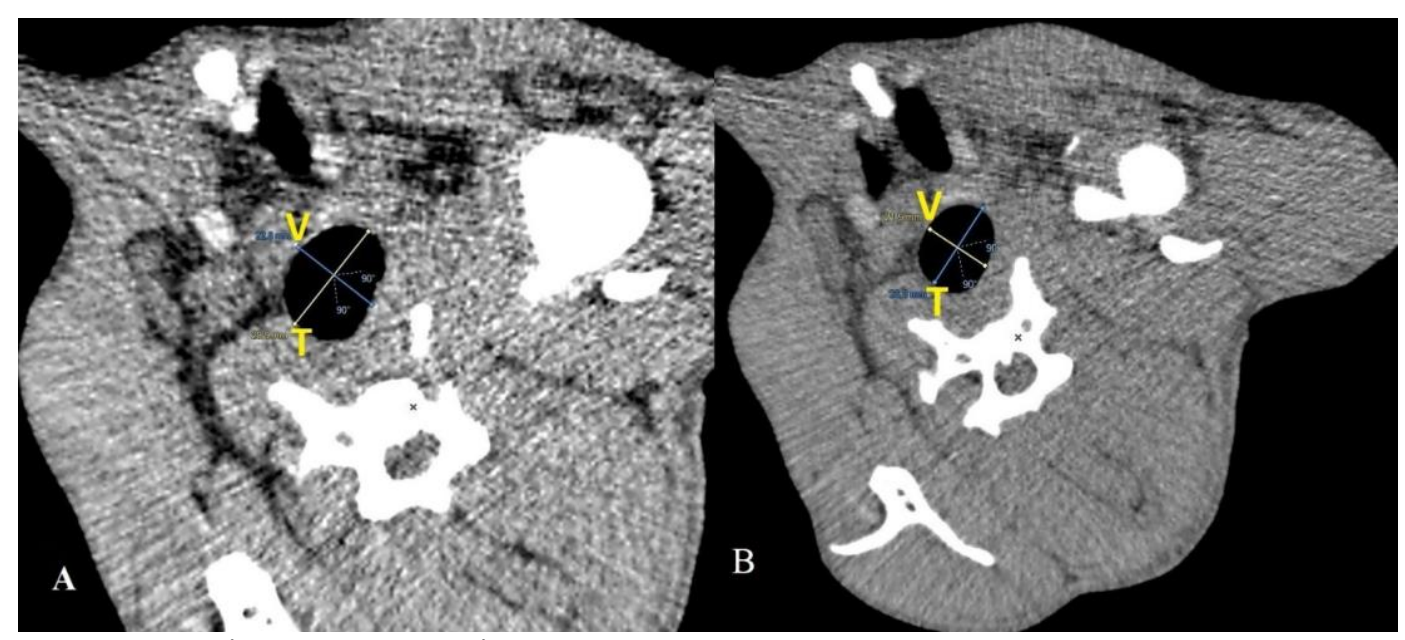

Şekil 2. Trachea'nın pars cervicalis'inin 2D transversal kesitleri ; A: Cranial nokta; B: Caudal nokta; T: Transversal çap; V: Vertical çap.

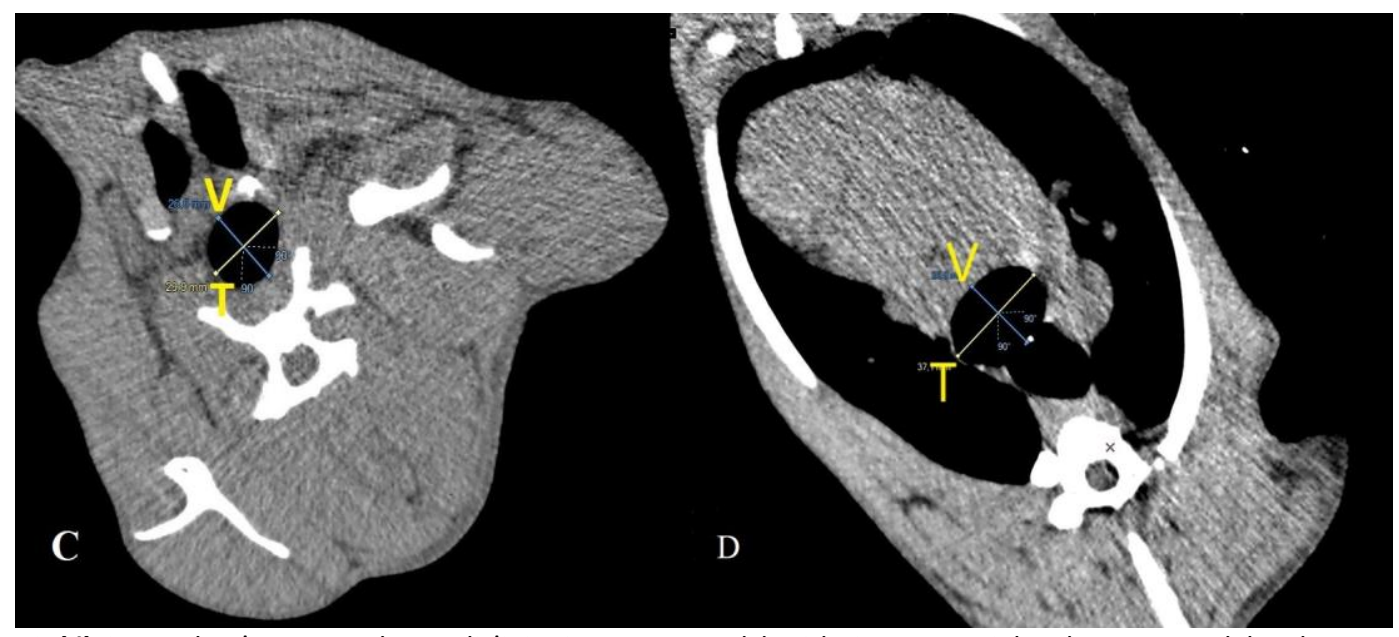

Şekil 3. Trachea'nın pars thoracalis'inin 2D transversal kesitleri ; C: Cranial nokta; D: Caudal nokta; T: Transversal çap; V: Vertical çap.

Cervical bölgede; vertical ve transversal çaplar ile CSA değerlerinde, cranial ve caudal noktalar arasında istatistiki açıdan farklılık bulunmamıştır. Ancak aynı noktalarda T/V oranında önemli bir farklılık $(P<0,001)$ tespit edilmiştir. Thoracal bölgede transversal çap, CSA ve T/V oranının caudal nokta değerlerinin, cranial nokta değerlerinden istatistiki olarak önemli derecede yüksek $(P<0,001)$ olduğu gözlemlenmiştir. Vertical çap değerleri açısından ise cranial ile caudal noktalar arasında önemli bir fark saptanmamıştır. Ölçüm yapılan bütün noktalardan alınan veriler ve üç boyutlu (3D) modelleme ile 
trachea'nın genel şeklinin hafif dorsoventral basık, tam olmayan elipsoid bir şekilde göründüğü ortaya konmuştur.

\section{Tartışma ve Sonuç}

Bazı araştırmacılar (Coyne ve Fingland, 1992; Fingland ve ark., 1995; Nelson, 1993; Salisbury ve ark., 1990; Smith ve ark., 1990; Watt, 1992; White ve Williams 1994) köpeklerde tracheal daralmayı değerlendirmek için radyografiyi kullanırken; Johanson ve ark. (1993) ile Van Pelt (1988) endoskopiden yararlanmıştır. Rudorf ve ark. (1997) ise bu amaç için ultrasonografiyi tercih etmiştir. Endoskopik yöntemde, tracheal değerlendirmeler için her ne kadar hassas teknik olarak görülse de, ölçümlerin hassas olmaması ve teknik olarak zor olması bir handikap olarak rapor edilmiştir (Johnson ve ark., 1993). Bazı yazarlar (Hedlund, 1984; Shih ve ark., 1997) ultrasonografide yüksek bir tecrübenin gerekliliğine işaret etmişlerdir. Fike ve ark. (1980) ile Walter (1999) bilgisayarlı tomografi görüntülemelerinin konvensiyonel tarama grafisindeki görüntülerden daha net olduğunu ortaya koymaktadır. Gerek hassas ölçümler gerekse elde edilen görüntülerin yüksek kalitesi, bu yönde bulgular sunan literaratürleri desteklemektedir.

Tracheal hava sütununun ölçümlerinin değerlendirilmesinde; hata payının az olması ve çarpık sonuçlarının düzeltilmesi yönüyle, en güvenilir metot olarak bilgisayarlı tomografi karşımıza çıkmaktadır (Griscom ve Wohl, 1986). Bu sebepledir ki beşeri hekimlikte trachea'nın BT ölçümleri, rutin bir tanı aracı olarak kullanılmaktadır (Chen ve ark., 2001; Mori ve ark., 2001; Roditi ve Weir, 1994; Shamberger ve ark., 1991). Huber ve ark. (1997) veteriner hekimlikte tracheal ölçümlerde BT kullanımının güvenilirliğinin test edilmediğini belirtmektedir. Kangal köpeklerinde yapmış olduğumuz ölçümlerin oldukça hassas olarak kaydedilebilmesi, bu yönü ile metodun sonuçlarının güvenilir olduğunu, ancak; köpeklerin solunumunun kontrolümüzün dışında kalması sebebi ile hata paylarının da olabileceğini göstermektedir. Alman çoban köpeklerinde yapılan bir çalışmada; trachea'nın cervical bölgesinde; vertical, transversal çaplar ve enine kesit alanı (CSA)'nın caudal nokta değerlerinin, cranial nokta değerlerine göre önemli ölçüde düşük bulunduğu bildirilmektedir (Kara ve ark., 2001). Araştırmamız, Kangal köpeklerinde bu değerlerin rakamsal olarak düşük olması ile beraber istatistiki olarak da önemli olduğunu göstermektedir.

Kara ve ark. (2001) T/V oranlarında ve thoracal bölge ölçümlerinde, istatistiksel farklılık olmadığını ifade etmişlerdir. Çalışma sonuçlarımızda ise cervical bölgede cranial nokta değeri, caudal nokta değerinden önemli derecede istatistiki yükseklik ortaya koymaktadır. Yine araştırmamızdan elde ettiğimiz bulgulara göre, trachea'nın thoracal bölümünde, vertical çap hariç diğer tüm ölçümlerin caudal nokta değerleri anlamlı şekilde yüksek bulunmuştur. Bu çalışmada elde edilen üç boyutlu görüntüler ve trachea'nın yeniden modellenmesi neticesinde, organın tam olmayan elipsoid bir yapıda olduğu ortaya konulmuştur. Bu durum, diğer birçok köpek ırkı ile benzerlik göstermektedir (Dyce ve ark., 2018).

Sonuç olarak, üç boyutlu bilgisayar tomografisi ile Kangal köpeklerinde yapılan bu çalışmada trachea'nın yapısı ve morfometrik değerleri belirlenmiştir. Elde edilen verilerin anatomi literatürüne ve veteriner hekimlik eğitimine katkı sağlayacağı düşünülmektedir.

\section{Kaynaklar}

Anonim,2017:https://www.researchgate.net/publication/ 263468806_Turkish_Kangal_Karabash_Shepherd_D og_in_English/, Erişim tarihi; 23.03.2017.

Atalar Ö, Koç M, Yüksel M, Arkaş AA, 2017: Kangal köpeklerinde pelvis boşluğunun bilgisayarlı tomografi ile üç boyutlu değerlendirilmesi. F Ü Sağ Bil Vet Derg, 31, 105-109.

Brovida C, Castagnaro M, 1992: Tracheal obstruction due to an eosinophilic granuloma in a dog, surgical treatment and clinicopathological observations. J Am Anim Hosp Assoc, 28, 8-12.

Burk RL, Ackerman N, 1996: Small Animal Radiology and Ultrasonograph. A Diagnostic Atlas and Text. Philadelphia, Saunders.

Carlisle CH, Bilry DN, Thrall DE, 1991: Tracheal and laryngeal tumors in the dog and cat, literature review and 13 additional patients. Vet Radiology, 32, 229-235.

Chen JD, Shanmuganathan K, Mirvis SE, Kileen KL, Dutton RP, 2001: Using CT to diagnose tracheal rupture. $A m$ J Roentgenol, 176, 1273-1280.

Coyne BE, Fingland RB, 1992: Hypoplasia of the trachea in dogs. J Am Vet Med Assoc, 201, 768-772.

Dallman MJ, Brown EM, 1984: Statistical analysis of selected tracheal measurements in normal dogs and dogs with collapsed trachea. Am J Vet Res, 45, 10331037.

Dyce KM, Sack WO, Wensing CJG, 2018: Köpek ve Kedide Thorax. In: Hazıroğlu RM, Çakır A. (Editors). Veteriner Anatomi Konu Anlatımı ve Atlas 4. Baskı, Güneş Kitabevleri: Ankara.

Dursun N, 1996: Veteriner Anatomi II. 3. Baskı, Medisan Yayınevi, Ankara.

Evans HE, 1993: Skeleton. In "Mille's Anatomy of the Dog", Ed; Evans HE, Philadelphia, USA.

Fike JR, Druy EM, Zook BC, Davis DO, Thompson JE, Chaney E, Bradley EW, 1980: Canine anatomy as assessed by computerized tomography. AmJ Vet Res, 41, 1823-1832.

Fingland RB, Layton Cl, Kennedy GA, Galland JC, 1995: A comparison of simple continuous versus simple 
interrupted sutura patterns for tracheal anastomosis after large-segment trachea1 resection in dogs. Vet Surg, 24, 320-330.

Getty R, 1975: The Anatomy of the Domestic Animals. 5nd Ed., Rosenbaum CE, Hillmann D, Philadelphia, USA.

Griscom NT, Wohl ME, 1986: Dimensions of the growing trachea related to age and gender. Am J Roentgelıol, 146, 233-237.

Hedlund CS, 1984: Tracheal anastomosis in the dog comparison of two end-to-end techniques. Vet Surg, 13, 135-142.

Huber ML, Henderson RA, Finn-Bodner S, Macintire DK, Wright JC, Hankes GH, 1997: Assesment of current techniques for determining tracheal luminal stenosis in dogs. Am J Vet Res, 58, 1051-1054.

Johnson LR, Krahvinkel DJ, Kiernan BC, 1993: Surgical management of atypical lateral tracheal collapse in a dog. J Am Vet Med Assoc, 203, 1693-1696.

Kara ME, Karaman ZC, Dabanoglu I, Ocal MK, 2001: Computed tomogaphic measurements of the trachea in dog. Dtsch Tierarztl Wschr, 108, 164-167.

Mori M, Ikemune MD, Hiasa H, 2001: Stenosis of the trachea caused by retrosternal ossification. Ann Thorac Surg, 71, 1035-1037.

Nelson WA, 1993: Lower Respiratory System. In: Slatter D (Editor) Textbook of Small Animal Surgery. Philadelphia: Saunders.

Nickel R, Schummer A, Seiferle E, 1979: The Anatomy of the Domestic Animals Volume 2, The Viscera of the Domestic Mammals. 2nd Revised edition by August Schummer, Richard, Wolfgang Otto Sack, Heidelberg,Berlin.

Nomina Anatomica Veterinaria, 2012: Prepared by the International Committes on Veterinary Gross Anatomical Nomenclature and Authorized by the General Assambly of the World Association of Veterinary Anatomists, The Editorial Committee Hannover, Sapporo, Japan.

Roditi GH, Weir J, 1994: The association of tracheomegaly and bronchiectasis. Clin Radiol, 49, 608-611.
Röcken H, Röcken M, 1994: Foreign body in the bifurcation trachea in a dog. Berl Münch Tierarztl Wschr, 107, 121-123.

Rudorf H, Herrtage ME, White RAS, 1997: Use of ultrasonography in the diagnosis of tracheal collapse. J Small Anim Prac, 38, 513-518.

Salisbury SK, Forbes S, Blevins WE, 1990: Peritracheal abscess associated with tracheal collapse and bilateral laryngeal paralysis in a dog. J Am Vet Med Assoc, 196, 1273-1275.

Shamberger RC, Holzman RS, Griscom T, Tarbell NJ, Weinstein HJ, 1991: CT quantitation of tracheal cross-sectional area as a guide to the surgical and anesthetic management of children with anterior mediastinal masses. J Pediatr Surg, 26, 138-142.

Shih JY, Lee LN, Wu HD, Yu CJ, Wang HC, Chang YL, Yang PC, 1997: Sonographic imaging of the trachea. J Ultrasound Med, 16, 773-790.

Smith MM, Gourley IM, Amis TC, Kurpershoek C, 1990: Management of tracheal stenosis in a dog. J Am Vet Med Assoc, 196, 931-934.

Van Pelt RW, 1988: Confirming tracheal hypoplasia in huskymix pups. Vet Med, 83, 266-273.

Walter PA, 1990: Alternative Imaging Technologies Computed Tomography. In: Lavin LM (Editor). Radiography in Veterinary Technology. Philadelphia: Saunders.

Watt PR, 1992: Congenital tracheal collapse in a young fox terrier. Aust Vet Pract, 22, 112-116.

White RAS, Williams JM, 1994: Tracheal collapse in the dog is there really a role for surgery? I Small Anim Prac, 35, 191-196.

Yılmaz O, 2017: Turkish Kangal (Karabash) shepherd dog (in English). https: //www.researchgate.net/ publication/ 263468806, Erişim tarihi; 23.03.2017.

*Yazışma Adresi: Ömer ATALAR

Fırat Üniversitesi Veteriner Fakültesi Anatomi Anabilim

Dalı, Elazığ- Turkey.

E-mail: atalar@firat.edu.tr 